\title{
A Simulation Study On Tune Modulation Effects in $\mathrm{RHIC}$
}

\author{
W. Fischer and T. Satogata
}

August 3, 1996

\begin{abstract}
Power supply ripple and momentum amplitudes together with a nonzero chromaticity are two important sources of tune modulation. Here, their influence on the dynamic aperture of RHIC is estimated at the proton injection energy. Element-by-element tracking is used to determine chaotic and loss borders. For this study, the object-oriented version of the tracking code TEAPOT has been modified to allow for time varying focusing strength, thereby creating tune modulation in the simulation.
\end{abstract}

\section{Introduction}

Nonlinear resonances can be excited in the presence of magnetic field errors. With tune modulation new resonances, called sidebands, are generated for each of these original resonances. The interaction of those new resonances among each other and with the original resonances can lead to a significantly larger chaotic region in phase space and to a reduced dynamic aperture $[1,2]$.

Through power supply ripple, multiples of the electrical ac net frequency are introduced as tune modulation frequencies. Momentum amplitudes together with a nonzero chromaticity create tune modulation with the synchrotron frequency. Ground motion and mechanical vibrations (for example caused by turbo vacuum pumps) give rise to low tune modulation frequencies. Only the first two sources are considered in this study.

The effect of tune modulation on the stability of particle motion can be estimated by element-by-element particle tracking. The loss borders and chaotic borders are determined without and with tune modulation. Different modulation depths are tested. The $\mathrm{C}++$ version of the tracking code TEAPOT [3] has been modified for this study.

\section{Tune Modulation in TEAPOT}

Early in 1996, Nikolay Malitsky of Cornell produced a C++ object-oriented tracking engine for TEAPOT that uses an SMF (Standard Machine Format) [4] lattice representation. This representation is flat - individual base optics elements occur in sequence but it is also hierarchical, including main magnet buses by name. 
Table 1: Quadrupole current modulation frequencies and amplitudes.

\begin{tabular}{|c|c|}
\hline Frequency $[\mathrm{Hz}]$ & $(\Delta I / I)_{\text {quad }}$ \\
\hline \hline 60 & $1.0 \cdot 10^{-7}$ \\
\hline 120 & $1.0 \cdot 10^{-7}$ \\
\hline 360 & $1.0 \cdot 10^{-7}$ \\
\hline 720 & $2.0 \cdot 10^{-6}$ \\
\hline 1440 & $4.0 \cdot 10^{-7}$ \\
\hline 2160 & $1.2 \cdot 10^{-7}$ \\
\hline
\end{tabular}

For tune modulation, approximately 10 lines of the original $\mathrm{C}++$ tracking engine code were modified. A new class, TeapotElemMod, was added that represents a single modulation of a single named lattice element or bus, and this class is used to describe each ripple line. The existing interface allows individual named elements (magnets and buses) to be modulated with single-frequencies, either in $\mathrm{C}++$ or in Perl. Future versions will add the ability to modulate each of several buses or magnets with several frequencies, including phase modulation to simulate transmission line effects.

The performance is optimized by precomputing arrays of modulation strengths before tracking. The modulation period is therefore restricted to integer numbers of machine turns. Tests on a Sparc Ultra give Teapot turn-by-turn tracking speeds of approximately $63 \mathrm{~ms} /$ turn with no modulations, and $83 \mathrm{~ms} /$ turn (a $30 \%$ increase) with modulation introduced by a single quadrupole.

\section{Simulated Machine Conditions}

A lattice of the RHIC Blue ring (in which particles travel clockwise) at the proton injection energy (relativistic $\gamma=27$ ) is used. The closed orbit distortions have rms values of 0.15 $\mathrm{mm}$ and $0.19 \mathrm{~mm}$ in the horizontal and vertical plane respectively. Tunes are set to $\left(\nu_{x}, \nu_{y}\right)=(18.19,19.18)$. The chromaticities $\Delta \nu /(\Delta p / p)$ are adjusted to +2 in both planes. Multipole errors are introduced as random numbers with the expected standard deviations and a cut at $3 \sigma$. The circumferential RF voltage is $88.2 \mathrm{MV}$ and the momentum amplitude $\Delta p / p$ is 0.001 .

Chromaticity and RF voltage lead to a tune modulation with the synchrotron frequency of $512 \mathrm{~Hz}$ and $2 \cdot 10^{-3}$ depth. A modulation spectrum for the main quadrupole bus is obtained in the following way [5]. A voltage spectrum is measured at the quadrupole power supply on a load resistor of $3.8 \mathrm{~m} \Omega$ at $300 \mathrm{~A}$ (the injection current is $550 \mathrm{~A}$ ). A regulator is expected to reduce subharmonics of the main frequency of $720 \mathrm{~Hz}$ by up to $40 \mathrm{~dB}$. As a conservative value we assume only $20 \mathrm{~dB}$ for this regulator. From measurements of a single quadrupole the transmission line characteristics of the main quadrupole bus are modeled. Together with the measured voltage ripple and the assumed active filter for subharmonics of the main frequency, modulation frequencies and amplitudes for the current are obtained. Six major lines can be identified which are listed in Tab. 1. All these 


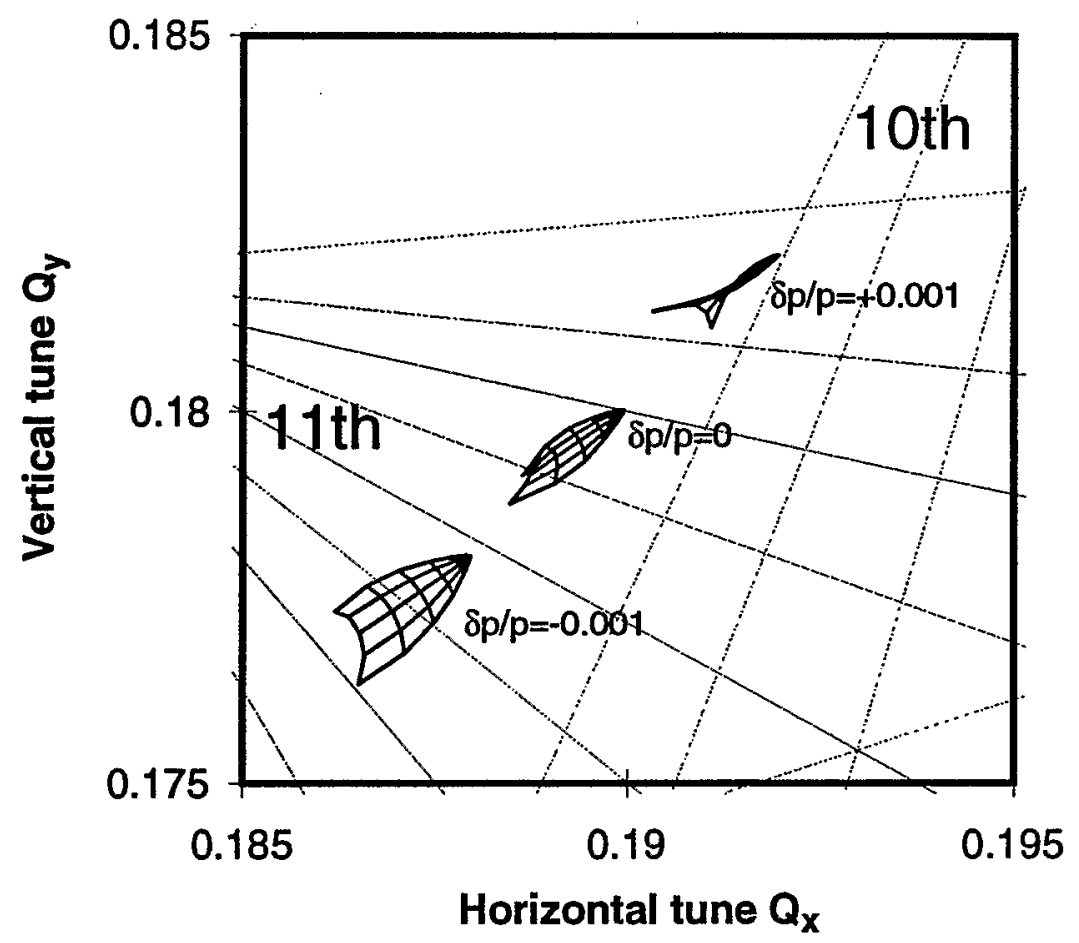

Figure 1: Tune space covered by a beam extending up to $6 \sigma$ in both transversal dimension.

lines have modulation depths significantly smaller than the modulation depth introduced through the synchrotron motion.

\section{Simulation Results}

Particles are tracked for 1000 turns turns to obtain the amplitude dependent tune. Amplitudes from 1 to $6 \sigma$, in steps of $1 \sigma$, and 5 different amplitude ratios are tested. A $\sigma$ of $2.24 \mathrm{~mm}$ at $\beta=50 \mathrm{~m}$, the maximum value for the beta function in the arcs, is assumed for both planes. The result is shown in Fig. 1 in the form of "tune leafs" for three different momenta. For the chosen working point no resonances below order 10 are relevant.

Loss and chaotic borders are determined after 10,000 turns ( $0.12 \mathrm{~s}$ storage time). Seven different amplitude ratios are tested. To obtain the chaotic border particle pairs are launched with an initially small phase space distance of $10^{-10} \mathrm{~m}$. Regular particle motion leads to a linearly increasing phase space distance with time while chaotic motion manifests itself in an exponential increase. Experience shows that particles that are found regular after $N$ turns, are usually not lost after $10 \times N$ turns. For this analysis the SIXTRACK post processor is used $[6,7]$.

Fig. 2 shows chaotic and loss borders without RF (and therefore without tune modula- 


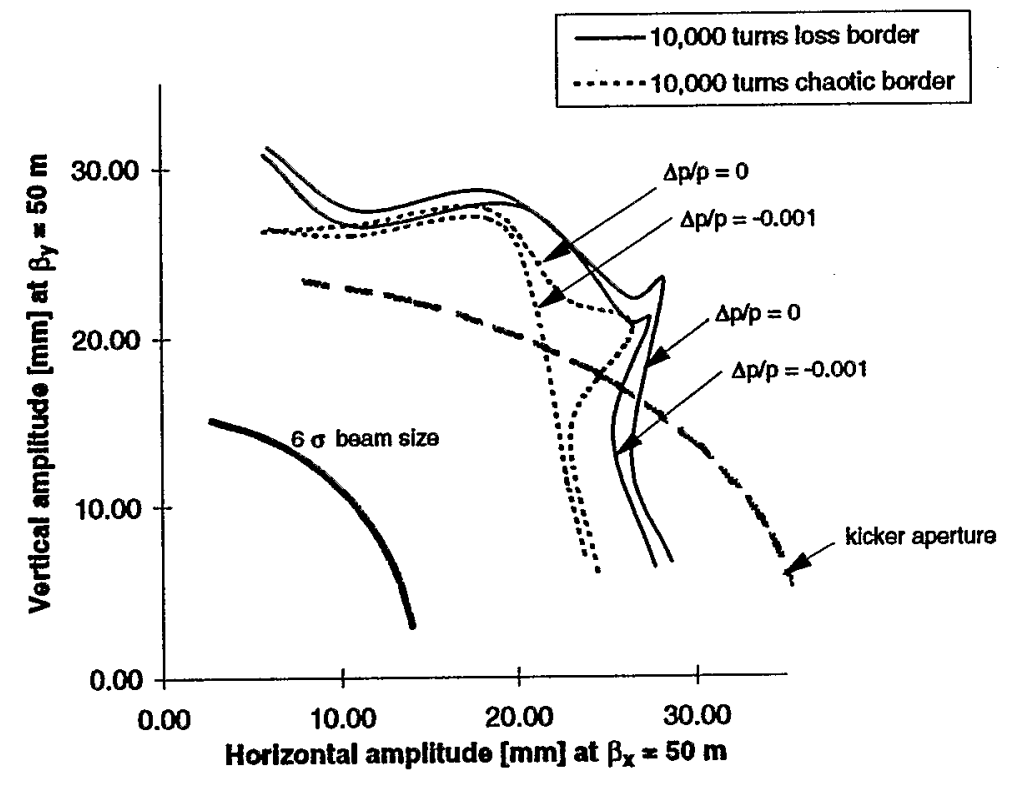

Figure 2: Loss and chaotic borders without RF and without power supply ripple for two different momentum deviations.

tion due to synchrotron motion) and without tune modulation due to quadrupole power supply ripple. Chaotic and loss borders for off-momentum particles are insignificantly smaller than those for on-momentum particles. In all cases the stability border is far outside the $6 \sigma$ beam limit and in half of the quadrant even outside the physical aperture of the injection kicker, which is the most limiting element in the ring in the absence of collimators.

Fig. 3 depicts the influence of tune modulation on the 10,000 turns loss border. In this and the following figure the term "ripple" denotes quadrupole power supply ripple. The multipliers (" $1 \times$ ", " $10 \times$ ", etc.) refer to the modulation depths given in Tab. 1. For comparison with Fig. 4 (discussed later) one chaotic border is also given. The cases "no ripple" (tune modulation due to synchrotron motion only), "ripple" and "10 $\times$ ripple" show practically no difference in the loss border. However, all these loss borders are somewhat smaller than the loss borders without RF (cf. Fig. 2). A further slight decrease in the loss border becomes apparent with " $100 \times$ ripple". With " $1000 \times$ ripple" the loss border is clearly reduced. In this case the modulation depths due to the power supply ripple are comparable to the modulation depth due to the synchrotron motion. The distorted loss and chaotic border in the region of smaller vertical amplitudes was observed in earlier studies to have a larger smear [8].

In Fig. 4 the 10,000 turns chaotic borders are depicted together with the loss border for the "no ripple" case. Again, there is practically no difference for the cases without, with and with 10 times modulation depth. However, all chaotic borders are significantly 


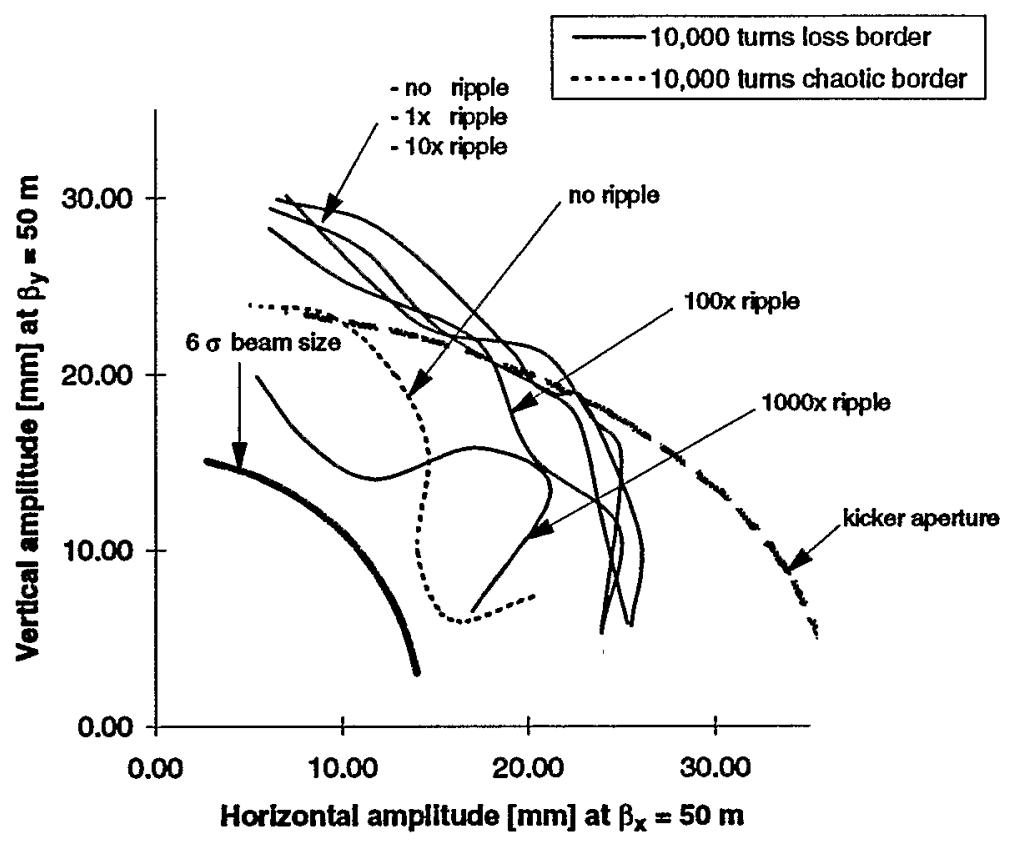

Figure 3: Loss borders with RF and quadrupole power supply ripple. The numbers in front of "ripple" refer to the modulation depth in Tab. 1. For comparison, one chaotic border is given.

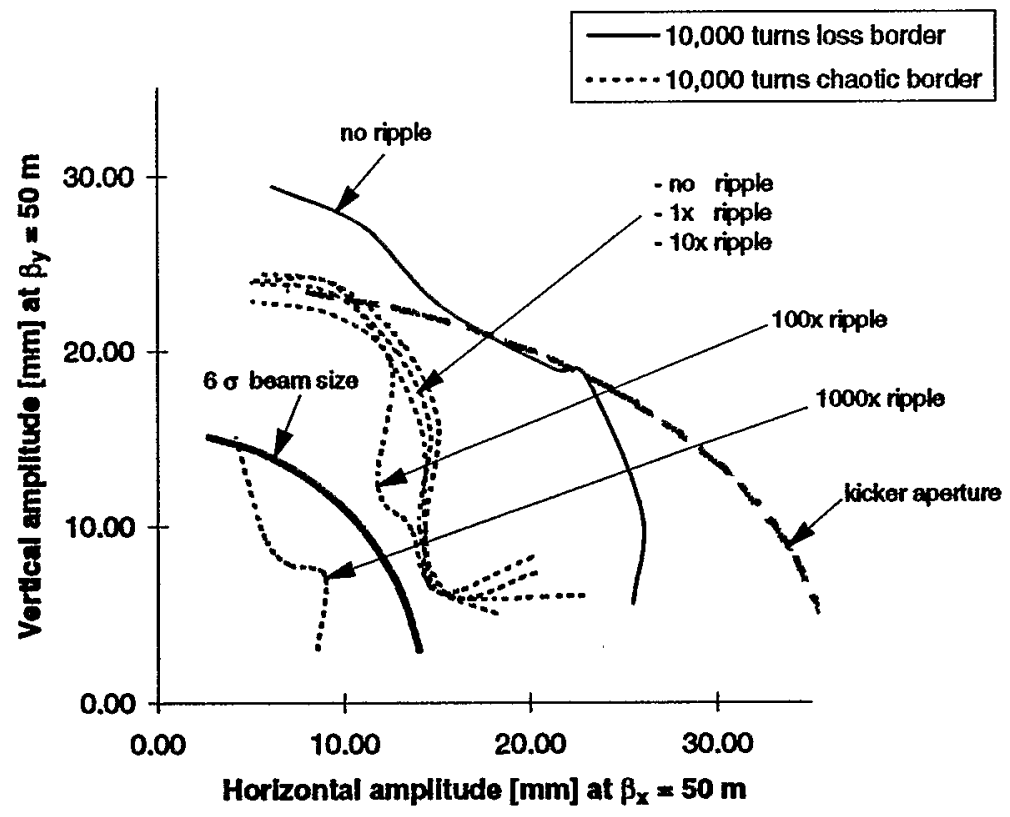

Figure 4: Chaotic borders with RF and quadrupole power supply ripple. The numbers in front of "ripple" refer to the modulation depth in Tab. 1. For comparison, one loss border is given. 
smaller than those without RF (cf. Fig. 2). A further reduced chaotic border is observed for the " $100 \times$ ripple" case and a drastically reduced chaotic border for the " $1000 \times$ ripple" case which lies even inside the $6 \sigma$ beam border.

As expected, the chaotic border is smaller than the loss border in all cases. However, it is known that, in particular with tune modulation, the chaotic border is a too pessimistic estimate for the dynamic aperture [2,9]. We therefore expect no dynamic aperture problems under the simulated conditions for at least $1.2 \mathrm{~s}(10 \times$ the tracked storage time) and modulation depth due to power supply ripple as large as 100 times the depths given in Tab. 1.

\section{Conclusion}

Our study indicates that no performance degradation is expected with the assumed tune modulation frequencies and amplitudes from power supply ripple at the proton injection energy. Further studies should also include low frequencies expected from ground motion or mechanical vibrations. Both, the injection energy for heavy ions and the top energy should be tested. At top energy the ripple of the power supplies for the interaction region quadrupole triplets will be important.

We are thankful to N. Malitsky for his support in the work on TEAPOT and to F. Dell for his assistance in producing a lattice model.

\section{References}

[1] T. Satogata, "Nonlinear resonance islands and modulational effects in a proton synchrotron", PhD Thesis, Northwestern University (1993).

[2] W. Fischer, "An Experimental Study on the Long-term Stability of Particle Motion in Hadron Storage Rings", PhD thesis Hamburg University, DESY 95-235 (1995) and CERN SL/96-10 (AP) (1996).

[3] L. Schachinger and R. Talman, "Manual for the program TEAPOT, noninteractive FORTRAN version" (1995); N. Malitsky, private communication (1996).

[4] N. Malitsky et. al., "A proposed flat yet hierarchical accelerator lattice object model", RHIC/AP/74 (1995).

[5] D. Bruno, B. Lambiase and C. Schultheiss, private communication (1996). 
[6] W. Fischer, "Transforming TEAPOT output into SIXTRACK postprocessing input", RHIC/AP/89 (1996).

[7] F. Schmidt, "SIXTRACK, version 1.1, single particle tracking code treating transverse motion with synchrotron oscillations in a symplectic manner", CERN SL/90-52 (AP) (1990).

[8] F. Pilat, private communication (1996).

[9] F. Schmidt, private communicatio (1995). 


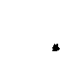

